\title{
Development of vortex state in circular magnetic nanodots: Theory and experiment
}

\author{
J. Mejía-López, ${ }^{1,2}$ D. Altbir, ${ }^{2,3}$ P. Landeros, ${ }^{4}$ J. Escrig, ${ }^{2,3}$ A. H. Romero, ${ }^{5}$ Igor V. Roshchin,,${ }^{6,7}$ C.-P. Li, ${ }^{8}$ M. R. Fitzsimmons, ${ }^{9}$ \\ X. Batlle, ${ }^{10}$ and Ivan K. Schuller ${ }^{8}$ \\ ${ }^{1}$ Facultad de Física, Pontificia Universidad Católica, Avenida Vicuña Mackenna 4860, Santiago, Chile \\ ${ }^{2}$ Center for the Development of Nanoscience and Nanotechnology (CEDENNA), 917-0124 Santiago, Chile \\ ${ }^{3}$ Departamento de Física, Universidad de Santiago de Chile (USACH), Avenida Ecuador 3493, 917-0124 Santiago, Chile \\ ${ }^{4}$ Departamento de Física, Universidad Técnica Federico Santa María, Avenida España 1680, Casilla 110-V, 2340000 Valparaíso, Chile \\ ${ }^{5}$ Materials Department, CINVESTAV, Querétaro, Mexico \\ ${ }^{6}$ Department of Physics and Astronomy, Texas A\&M University, 4242 TAMU, College Station, Texas 77843-4242, USA \\ ${ }^{7}$ Materials Science and Engineering Program, Texas A\&M University, 3003 TAMU, College Station, Texas 77843-3003 USA \\ ${ }^{8}$ Department of Physics, University of California-San Diego, La Jolla, California 92093-0319, USA \\ ${ }^{9}$ Los Alamos National Laboratory, Los Alamos, New Mexico 87545, USA \\ ${ }^{10}$ Departament de Fisica Fonamental, Universitat de Barcelona, 08028, Barcelona, Catalonia, Spain
}

(Received 30 December 2009; revised manuscript received 9 April 2010; published 18 May 2010)

\begin{abstract}
We compare magnetic reversal of nanostructured circular magnetic dots of different sizes. This comparison is based on superconducting quantum interference device (SQUID) magnetometry, neutron scattering, Monte Carlo simulation, and analytical calculations and is quantified using a parameter which characterizes the variation in the hysteresis curve width. Below a critical dot diameter, the magnetic reversal occurs by coherent rotation and above that diameter, the reversal occurs by formation of a magnetic vortex. The vortex-core diameter is controlled by competing magnetic energy contributions. For 20-nm-thick Fe dots, the values of the critical diameter $(58-60 \mathrm{~nm})$ and the vortex core $(16-19 \mathrm{~nm})$ are in very good agreement between the different experimental and theoretical methods: neutron scattering, SQUID magnetometry, Monte Carlo simulations, and analytical calculations.
\end{abstract}

DOI: 10.1103/PhysRevB.81.184417

PACS number(s): 75.75.Fk, 75.10.-b

\section{INTRODUCTION}

The interest in magnetic nanostructures has drastically increased in the past decade, mainly due to the great progress in experimental techniques and recent technological developments, which allow access to the nanometer length scales. In particular, understanding the behavior of solids in confined geometries at the nanoscale is an important area of basic research that produced many surprises especially in the field of nanomagnetism. ${ }^{1,2}$ Moreover, these types of nanostructured magnets may have relevance in a variety of spintronics and magnetic storage applications, including magnetic bitpatterned media and magnetic random access memory. One of the most studied types of such structures are nanoscaled disks denoted as "nanodots" which have been reproducibly fabricated by variety of techniques. ${ }^{3-6}$ In these magnetic dots, many different magnetic configurations appear and at a particular length scale, a magnetic vortex may become the ground state..$^{1,7,8}$ This is caused by an intricate balance between the different relevant energy contributions: anisotropy (surface, boundary, etc.), Zeeman, and exchange. Appearance and stability of the vortex also have important implications for the possible use of the "vorticity" as an information storage channel. To create such devices, it is important to characterize and understand the magnetic stability, coercivity, remanent field, etc., at short length scale. In particular, it is of great importance to understand the dependence of these properties on the controlling parameters, such as applied magnetic field and dot size.

A magnetic vortex in a nanodot is a spin configuration with an in-plane magnetic flux closure which appears during magnetization reversal, generally when the size is comparable to or smaller than the domain-wall width. Nucleation of the vortex is associated with the appearance of an out-ofplane magnetization, the "vortex core," caused by the inherent singularity developing from a circular flux closure. In response to the changing in-plane external field, the vortex propagates across the nanostructure, annihilating at a critical, "annihilation" field. ${ }^{9,10}$ Thus, after in-plane saturation, as the field is decreased the spins are able to orient out of plane, overcoming the in-plane shape anisotropy. The return to the in-plane configuration in the reversed in-plane saturated state may also exhibit very interesting physics. Therefore, it is important to understand how various parameters affect these transitions. It is also important to note, that this vortex state can also be excited and manipulated by various means, e.g., magnetic field or electric current. ${ }^{8,11-13}$

In this paper, after briefly describing recently reported experimental results, ${ }^{14}$ we discuss details of the theoretical studies the formation, properties, and stability of a single magnetic vortex as a function of dot size. The experimental studies are performed using superconducting quantum interference device (SQUID) magnetometry and small anglepolarized neutron scattering. ${ }^{14}$ These results are compared to those obtained by theoretical approaches. Monte Carlo simulations performed using a scaling technique ${ }^{15-18}$ provide the dependence of several properties within the hysteresis loop on the dot diameter and characterize the core diameter when the vortex is centered in the dot at remanence. To compliment the numerical simulations, we evaluate the core diameter analytically.

This paper is organized as follows. In Secs. II and III, we present a description of the experimental methodology and 


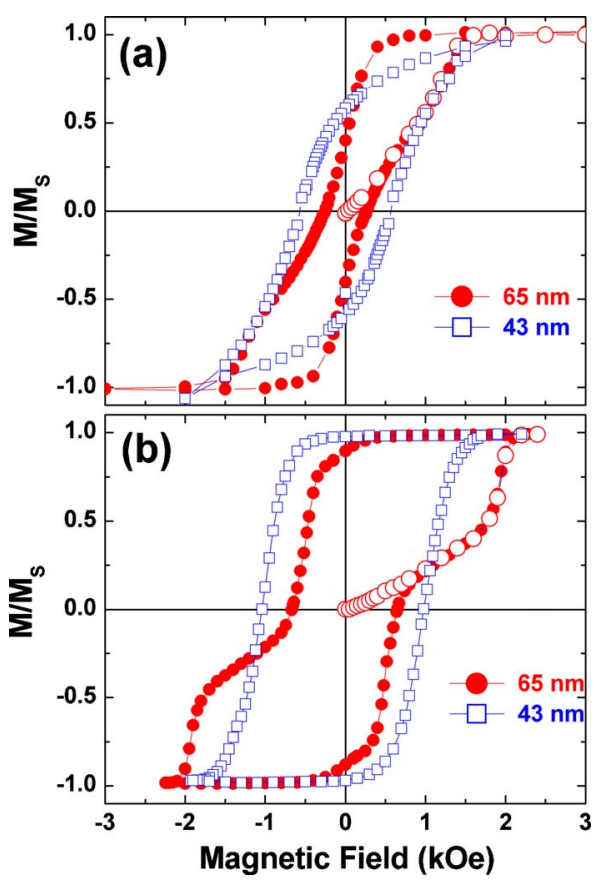

FIG. 1. (Color online) Hysteresis loops for 43- (squares) and 65(circles) nm-diameter, 20-nm-thick $\mathrm{Fe}$ dots at $10 \mathrm{~K}$, and virgin curve (empty circles) for the $65 \mathrm{~nm}$ dots: (a) experimental data (after Ref. 14); (b) Monte Carlo simulations for the same conditions.

the theoretical calculations, respectively. Then, we discuss the magnetization-reversal mechanisms and present a detailed analysis of the microscopic structure of the vortex core. Finally, we compare the theoretical results with the experimental ones. In Sec. IV, we summarize our findings.

\section{EXPERIMENT}

Arrays of sub-100 nm Fe nanodots are prepared using electron-beam evaporation combined with lift-off of anodized alumina masks on silicon substrates. ${ }^{19}$ Powder x-ray diffraction shows that the hexagonally ordered $\mathrm{Fe}$ dots are polycrystalline. By controlling the anodization process (i.e., electrochemical parameters) and using double anodization ${ }^{20}$ dot diameters and periodicities in the range $25-150 \mathrm{~nm}$ with narrow distributions (10-15 standard deviation) are produced. The dot periodicity is typically nearly twice the dot diameter.

The magnetic properties are determined from dc SQUID magnetometry and first-order reversal curves (FORC) between 10 to $300 \mathrm{~K}$ and small angle-polarized neutron scattering in the range of the out-of-plane wave vector transfer, $\mathrm{Q}_{z}$, between 0.005 and $0.015 \AA^{-1}$.

Figure 1 shows (a) experimental and (b) simulated hysteresis loops for magnetic dots with $20 \mathrm{~nm}$ height and diameter $D$ (for clarity, only two diameters, 43 and $65 \mathrm{~nm}$, are shown). For these sizes, the domain-wall width is comparable to the dot size; therefore, Fe dots with sub-100 $\mathrm{nm}$ diameters are not expected to form multidomain states. ${ }^{21}$ In this case, the coercivity and the equilibrium magnetic state depend on the

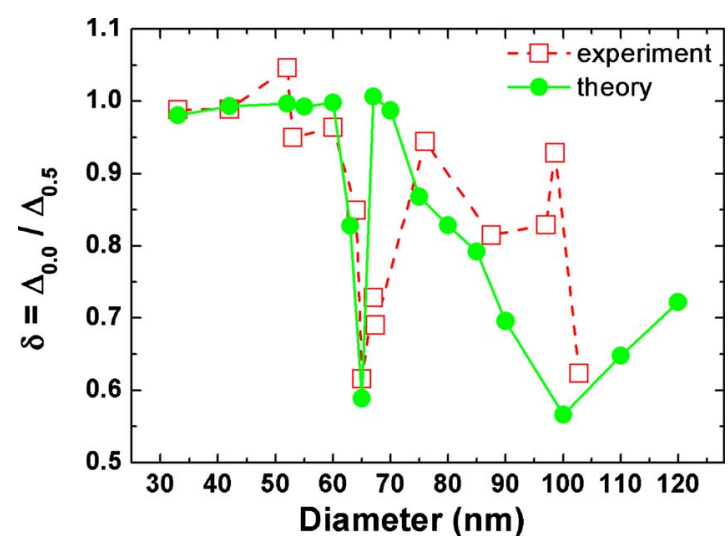

FIG. 2. (Color online) Ratio of the loop width at coercivity to the width at half saturation as a function of the dot diameter for 600 Monte Carlo steps (after Ref. 14).

dot diameter. For very small diameters, the hysteresis loops strongly resemble curves described by Stoner-Wohlfarth model for reversal of single-domain elements. ${ }^{22}$ This can be seen for the 43-nm-diameter dots. Dots with diameters larger than $\sim 60 \mathrm{~nm}$ show reduced coercivity, with a narrowing of the hysteresis loop close to the zero-magnetization states, as shown in Fig. 1 for the 65-nm-diameter dots.

To quantify the effects of dot size on the hysteresis loop shape, we introduce the parameter $\delta=\Delta_{0.0} / \Delta_{0.5}$, with $\Delta_{0.0}$ and $\Delta_{0.5}$ the widths of the hysteresis loop at $M=0$ (zero magnetization) and $M=0.5 M_{s}$ (half saturation), respectively, $M_{s}$ being the saturation magnetization. Figure 2 shows the dependence of $\delta$ on the dot diameter obtained from both the experimental loops (hollow red squares) and Monte Carlo simulations (full green circles), described below. For dot diameters around $65 \mathrm{~nm}$, this ratio is greatly reduced. This is typical for reversal via a vortex state as also observed with FORC measurements, ${ }^{23}$ confirmed by simulations ${ }^{9}$ and discussed in detail in the theoretical section below. The virgin curve measured from the as-grown state (never exposed to magnetic field) or after demagnetization by field cycling about minor hysteresis loops is almost linear in a large field range. For diameters larger than $60 \mathrm{~nm}$ it joins the major hysteresis loop (see Fig. 1). This very unusual behavior is in a good agreement with the results of Monte Carlo simulations. ${ }^{9}$

Below, we will focus on an array of Fe dots of average diameter of $65 \pm 7 \mathrm{~nm}$ with spacing of $110 \pm 12 \mathrm{~nm}$ and thickness of $20 \mathrm{~nm}$ covering a $\sim 1.8 \mathrm{~cm}^{2}$ (Fig. 3). Since for these dots (at the deep minimum in Fig. 2) the ground state is expected to be a vortex, we performed grazing incidence small angle neutron scattering with polarization analysis [polarized grazing incidence small-angle neutron scattering (GIS-ANS)] to measure the perpendicular magnetization, $M_{z}$, of the vortex core and its diameter ${ }^{14}$ For these measurements, the sample is magnetically conditioned using the following protocol. First, a small, 34 Oe, field is applied along the surface normal of the sample (along $+\hat{z}$ ) to set the preferred direction for the vortex-core magnetization (core polarity). Next, a second field of $-4 \mathrm{kOe}$ is applied in the sample plane (along $-\hat{y}$ ) to saturate the dots. Then the inplane field is slowly increased to +300 Oe-a field that ex- 


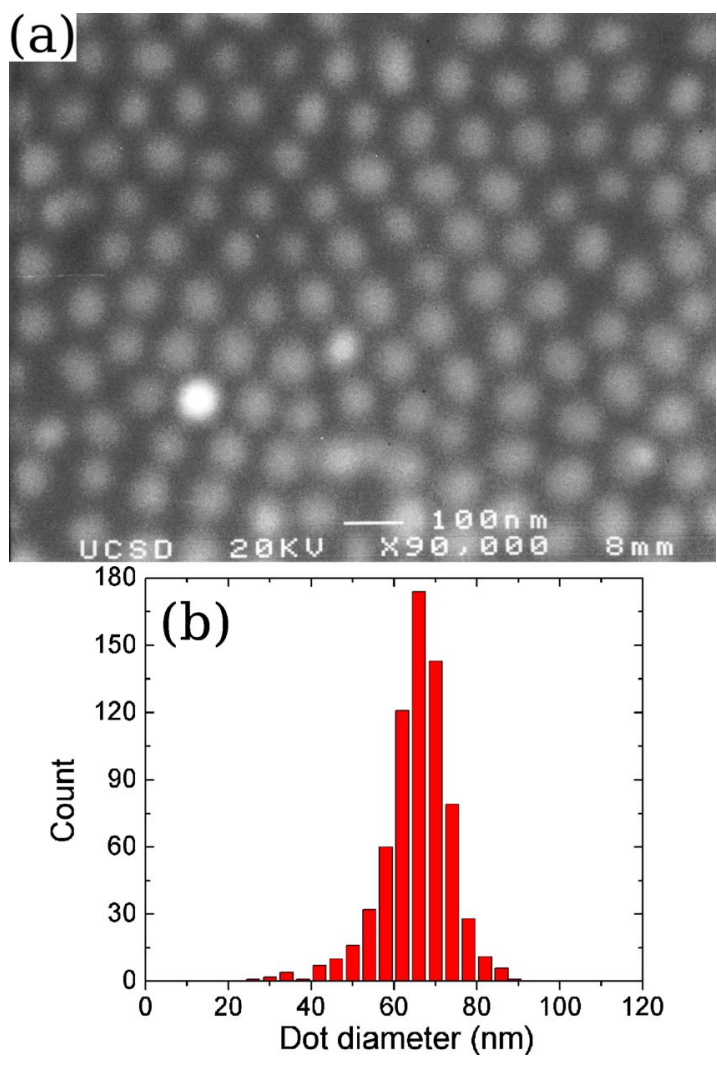

FIG. 3. (Color online) (a) Scanning electron microscope image of the sample fabricated for neutron-scattering experiment. (b) Distribution of the dot diameters. Average diameter $65 \pm 8 \mathrm{~nm}$.

ceeds the vortex nucleation field (Fig. 1) - before returning this field to zero, leaving only the 34 Oe out-of-plane field. ${ }^{24}$

These quantitative measurements yield the out-of-plane magnetization of the dot in the vortex state, i.e., the magnetization of the vortex core, $140 \pm 50 \mathrm{emu} / \mathrm{cm}^{3}$. An equivalent $\mathrm{Fe}$ cylinder with the same magnetization would have a diameter of $19 \pm 4 \mathrm{~nm}$. Very similar numbers are obtained by modeling the vortex core with a different, more realistic spatial distribution of spins (for instance, a circular Gaussian function, paraboloid, etc.).

\section{THEORETICAL CALCULATIONS}

The geometry implies that these dots can be modeled as noninteracting (i.e., without dipolar, exchange, or other couplings) due to their large separation. ${ }^{9,19,25-27}$ Earlier calculations have shown that when the side-to-side distance in an array is larger than a single-dot diameter (i.e., the center-tocenter distance larger than twice the diameter) the dots interact weakly. ${ }^{9}$ Since the dots are polycrystalline, we neglect the crystalline anisotropies. Thus, both in the Monte Carlo simulation and analytical calculations we use these assumptions as a starting point.

\section{A. Monte Carlo simulations}

Since the methods used here are standard, here we only review the main concepts used for the simulations. ${ }^{9}$ The total energy, $E_{t o t}$, of a single dot with $N$ magnetic moments is given as

$$
E_{t o t}=\frac{1}{2} \sum_{i \neq j}\left(E_{i j}-J_{i j} \hat{\mu}_{i} \cdot \hat{\mu}_{j}\right)+E_{H},
$$

where $E_{i j}$ is the dipolar energy as

$$
E_{i j}=\left[\vec{\mu}_{i} \cdot \vec{\mu}_{j}-3\left(\vec{\mu}_{i} \cdot \hat{n}_{i j}\right)\left(\vec{\mu}_{j} \cdot \hat{n}_{i j}\right)\right] / r_{i j}^{3}
$$

with $r_{i j}$ the distance between the magnetic moments $\vec{\mu}_{i}$ and $\vec{\mu}_{j}$ and $\hat{n}_{i j}$ the unit vector along the direction that connects the two magnetic moments. $J_{i j}$ is the exchange coupling, which is assumed nonzero only for nearest neighbors and $\hat{\mu}_{i}$ is a unit vector along the direction of $\vec{\mu}_{i} \cdot E_{H}=-\Sigma_{i} \vec{\mu}_{i} \cdot \vec{H}$ is the Zeeman energy.

Because of the large number of magnetic moments within each particle, a brute force calculation of the magnetic configuration of a 10-100 nm structure is unreachable with present standard computational facilities even using the above-mentioned phenomenological energy function. To avoid this problem we use a scaling technique developed by d'Albuquerque e Castro et al. ${ }^{15}$ in which the number of spins is reduced to a value suitable for numerical calculations. This procedure decreases the dipolar field exerted on a particle, and therefore the exchange coupling constant is scaled down to keep the correct balance between magnetostatic and exchange energies, responsible for domain formation and reversal mechanisms. With this procedure ${ }^{15,18}$ the magnetic properties of a nanoparticle of dimensions $d$ is equivalent to the one of a smaller particle with dimensions $d^{\prime}=d x^{\eta}$ being $x<1$ and $\eta \approx 0.55-0.57$, if the exchange constant is also scaled as $J^{\prime}=x J$. This scaling method, in combination with Monte Carlo simulations, ${ }^{16,18}$ provides the correct magnetic state of a single nanoparticle. This approach was tested using different values of the scaling parameter $x$ and the results were shown to be independent of it. ${ }^{15,18}$

This method applied to granular Fe, using $\left|\vec{\mu}_{i}\right|=\mu$ $=2.2 \mu_{B}$, a lattice parameter $a_{0}=2.8 \AA$ and $J=42 \mathrm{meV}$ (Ref. 28), produces a Curie temperature for bulk Fe of $1043 \mathrm{~K}$, in good agreement with textbook values. Here, we employ the scaling technique replacing, the magnetic dot by a smaller one with a scaling factor of $\eta=0.57 .{ }^{15,16,18}$ Correspondingly, we also scale the exchange interaction by a factor $x \equiv J^{\prime} / J$ $=2.1 \times 10^{-3}$, i.e., $J$ is replaced by $J^{\prime}=0.09 \mathrm{meV}$ in the expression for the total energy.

Monte Carlo simulations are carried out using the Metropolis algorithm with local dynamics and single spin-flip methods. ${ }^{29}$ The new orientation of the magnetic moment is chosen arbitrarily with a probability $p=\min [1, \exp ($ $\left.-\Delta E / k_{B} T\right)$ ], where $\Delta E$ is the change in energy due to the reorientation of the spin, $k_{B}$ is the Boltzmann constant, and $T$ is the temperature. For our simulations we use $T=10 \mathrm{~K}$, at which the experimental hysteresis loops are measured. The temperature has been scaled as $T^{\prime}=x T$, according to the description in Ref. 9.

For the simulation of magnetic hysteresis the number of Monte Carlo steps (MCS) used is a critical issue. We follow the procedure used by many authors ${ }^{29,30}$ in which the number of MCS is changed until a fair agreement with the experi- 


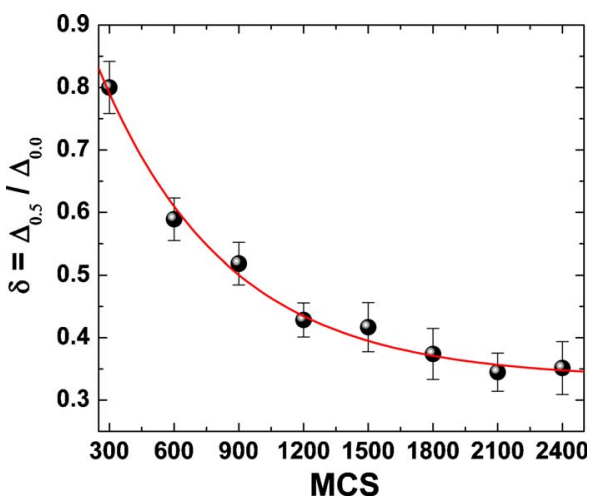

FIG. 4. (Color online) Ratio of the loop width at coercivity to the width at half saturation for a $65 \mathrm{~nm}$ diameter dot as a function of number of MCS.

mental results is obtained. Then, the number of MCS is kept fixed and all other variables are modified. Hence, we first study the effect of the number of MCS on $\delta$. Figure 4 illustrates $\delta$ for a $65-\mathrm{nm}$-diameter dot as a function of the number of MCS. $\delta$ asymptotically converges to 0.35 for $2400 \mathrm{MCS}$ per field value. However, the effects discussed here are weakly dependent on the MCS for MCS $\geq 600$.

In the simulation, the magnetization curve is started at $H=2.6 \mathrm{kOe}$ applied along the [100] crystallographic direction, labeled the $x$ axis, with the initial configuration in which most of the magnetic moments point along this direction. We define $M_{s}$ as the magnetization at the maximum applied field $(2.6 \mathrm{kOe}), M_{r}$-the remanent magnetization, and $H_{c}$-the coercivity. Field steps of $\Delta H=10$ Oe are used in all calculations. Typically, we perform $6.24 \times 10^{6}$ Monte Carlo steps per spin for a complete hysteresis loop, which is equivalent to $600 \mathrm{MCS}$ per field value. Forty different seeds are used for the random number generator to improve the statistics by considering different configuration states. These 40 simulations are averaged to generate the present results.

Two examples of simulated hysteresis loops are shown in Fig. 1(b), for the same diameters used in the experiment. The calculated loops are strongly dependent on the dot diameter and the coercivity exhibits a significant change as a function of dot size. To clarify this behavior, we investigate the magnetic configurations a dot exhibits going through its hysteresis loop, and we focus on the dependence of these magnetic configurations on the dot diameter. In general, magnetization reversal for considered here small-sized planar dots occurs by two main mechanisms, depending on the dot diameter. In the first one, known as "coherent rotation,"22 the spins follow the magnetic field orientation without formation of any complex magnetic structure inside the magnetic dot. In the second one, magnetization reversal occurs via displacement of a more complex spin structure such as a magnetic vortex, which nucleates at one side of the dot, moves across the dot, and annihilates on the other side. ${ }^{9}$

For a direct comparison to experimental observations, we study the magnetic behavior as a function of dot diameter using the same $\delta=\Delta_{0.0} / \Delta_{0.5}$ parameter to characterize the hysteresis (Fig. 2). The zero magnetization width $\delta$ changes dramatically, showing a complex dependence on dot diameter. For very small dots, the ground state is a single domain (a)

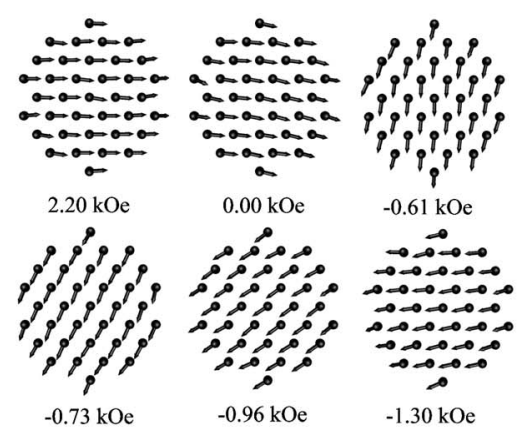

(b)

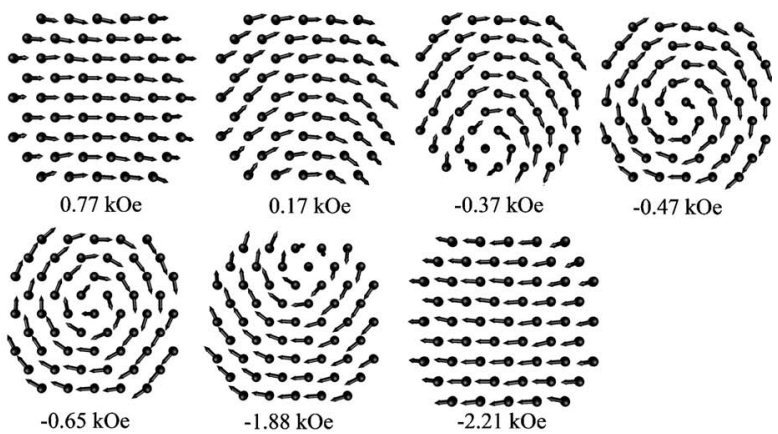

(c)

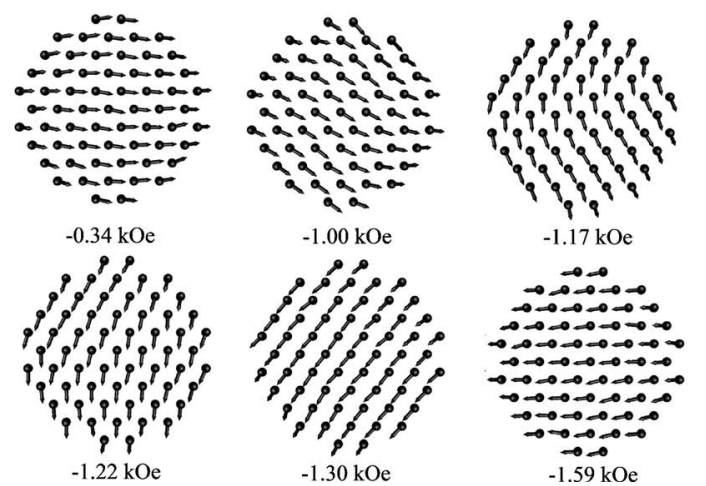

FIG. 5. Magnetic configurations during the switching process for different dot diameters: (a) $D=55 \mathrm{~nm}$, (b) $D=65 \mathrm{~nm}$, and (c) $D=67 \mathrm{~nm}$.

and its magnetization reverses coherently, exhibiting a hysteresis loop with a constant width, ${ }^{22}$ hence $\delta \approx 1$. When the diameter of the dot increases, vortex nucleation drives the reversal and a "neck" is observed at zero magnetization (see below). This effect is enhanced at $D=65 \mathrm{~nm}$, where $\delta$ $\approx 0.6$. While the precise $\delta$ value may depend (slightly) on the number of MCS (Fig. 4), this "best" diameter is independent of it. A fair agreement between theoretical and experimental hysteresis loops is obtained for $600 \mathrm{MCS}$, which also provides a very good agreement between the $\delta$ values obtained from experiments and simulations (Fig. 2).

Figure 5 illustrates snapshots of the spin configurations for different values of the applied magnetic field. For dots diameters smaller than $60 \mathrm{~nm}$, in all 40 simulations, these dots reverse their magnetization via the same mechanism: coherent rotation [Fig. 5(a)]. From 60 to $65 \mathrm{~nm}$, an increasing number of dots (from 1 to 14), among the 40 seeds used, reverse their magnetization through the nucleation of a vortex, which progressively decreases $\delta$. For $D=65 \mathrm{~nm}$, the reversal of all the dots occurs via the nucleation of a vortex state, as illustrated in Fig. 5(b). At this $D, \delta$ reaches a mini- 


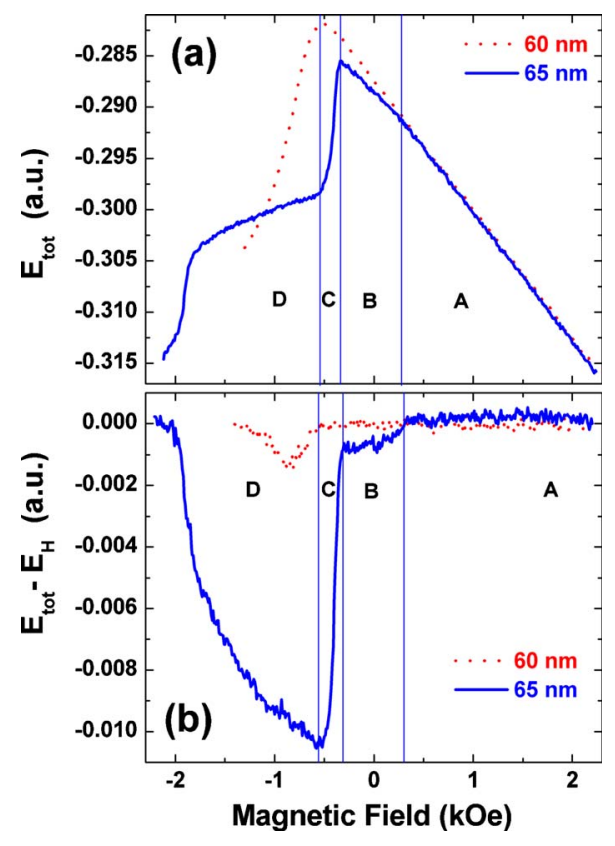

FIG. 6. (Color online) Energies during the field reversal for a 60 $\mathrm{nm}$ (dashed red line) and a $65 \mathrm{~nm}$ dot (continuous blue line): (a) total magnetic energy during the reversal, (b) internal energy. Letters identify different regimes as explained in the text. Internal energy has been shifted to zero value and expressed in arbitrary units.

mum. While magnetization reversal via vortex is the only reversal mode present for 65-nm-diameter dots, for larger dots, reversal occurs both via a vortex and an S state (coherent rotation) [Fig. 5(c)]. This leads to an increase in $\delta$ to almost one at $D=67 \mathrm{~nm}$, where reversal occurs primarily via coherent rotation. For $67<D<80 \mathrm{~nm}$, reversal for some of the dots again occurs via vortex, resulting in a new decrease in $\delta$. At $D=80 \mathrm{~nm}$, magnetization in all the dots reverses again via vortex nucleation. It is worth noting that this does not necessarily reflect that the states via which the reversal occurs are the ground states at zero field. For most of the dot diameters above $60 \mathrm{~nm}$ vortex is the ground state at zero applied field. Above $65 \mathrm{~nm}$, the vortex is probably unstable because the domains of out-of-plane magnetization may not be commensurate with the dot diameter, hence decreasing the gain in the total energy from nucleating a vortex. ${ }^{14}$

Figure 6 shows (a) total and (b) internal (total minus Zeeman) energies for two dot diameters: 60 and $65 \mathrm{~nm}$. In region A, all spins point along the applied field $H$ and the internal energy remains almost constant. In region $\mathrm{B}$, a small difference arises between the two dots. For $D=60 \mathrm{~nm}$, the spins are aligned along the magnetic field, whereas for $D$ $=65 \mathrm{~nm}$, a so-called $\mathrm{C}$ state is formed. This $\mathrm{C}$ state is a lower energy state and therefore there is an energy decrease, (Fig. 6). Region $\mathrm{C}$ shows an even larger difference between the two dot sizes. For the $60 \mathrm{~nm}$, the energy remains almost constant although some spins start canting due to the competition between the dipolar interaction and the applied reversal field. In contrast, in the $65 \mathrm{~nm}$, the formation of the vortex decreases the energy to a deep minimum, at the coercive field. This implies that a larger energy, and hence a large reversal magnetic field is needed for the magnetic configu- ration to return to a state where all spins are lined up along the applied field, as found in region D. This explains the neck formation in the hysteresis loop. The $60 \mathrm{~nm}$ dots on the other hand exhibit a small minimum around $-0.85 \mathrm{kOe}$ due to spin canting without vortex formation. The field corresponding to this energy minimum also coincides with the coercive field. The energy barrier to come out from this canted state is much smaller than that for the vortex state. In region $\mathrm{B}$, although the vortex is the ground state for the 65 nm dots, ${ }^{9}$ after in-plane saturation the dot may become stuck in the metastable $\mathrm{C}$ state due to the high-energy barrier between it and the vortex state. This explains the finite remanent magnetization and the nonzero coercivity. Thermal activation at higher temperatures may cause earlier vortex nucleation (i.e., at more positive fields) as found experimentally. ${ }^{31}$

To calculate the core size with a reasonable precision, it is necessary to increase the number of spins considered in our simulated dot. Therefore, for this calculation we choose $x$ $=0.00476$, which gives $J^{\prime}=0.2$, which sets $N=1332$.

The dot is magnetically prepared following a procedure similar to that in the neutron-scattering experiment. ${ }^{14}$ First, an external magnetic field of $3 \mathrm{kOe}\left(H_{x}\right.$ along the $+\hat{\mathbf{x}}$ direction) and $34 \mathrm{Oe}$ along the $+\hat{\mathbf{z}}$ direction, $H_{z}$, is applied. Then $H_{x}$ is reduced in steps of 10 Oe until the magnetization becomes almost zero, in order to stabilize the vortex state. This occurs when $H_{x}$ points along the negative direction of $\hat{\mathbf{x}}$. Finally, $H_{x}$ is increased to zero. It is important to note that for all the calculation $H_{z}$ is kept fixed. Using color coding for magnetization along the $z$ direction, Fig. 7(a) illustrates the vortex-core profile obtained with these Monte Carlo simulations. The total magnetization at $H_{x}=0$, after performing the procedure defined above, is $0.06 M_{s}$. The vortex-core size $R_{c}$ is calculated as in the experiment. We assume that the magnetization along the $\hat{\mathbf{z}}$ direction arises from spins totally saturated along the $+\hat{\mathbf{z}}$ direction inside a cylinder of the same height of the dot. Following these procedure, we find the vortex-core size of $R_{c}=R \sqrt{M / M_{s}} \sim 16 \mathrm{~nm}$ in quantitative agreement with experiment.

\section{B. Analytical calculations}

\section{Vortex core}

For an additional characterization of the vortex we employ a simplified description ${ }^{25,32}$ in which the discrete distribution of magnetic moments is replaced with a continuous one, described by the magnetization field $\vec{M}(\vec{r})$. With this $\vec{M}(\vec{r}) \delta v$ gives the magnetic moment within the volume $\delta v$ centered at $\vec{r}$. In this, "micromagnetic" approach, the total energy $\left(E_{t o t}\right)$ of a ferromagnet is given by the sum of three terms corresponding to the magnetostatic $\left(E_{\text {dip }}\right)$, exchange $\left(E_{e x}\right)$, and Zeeman $\left(E_{z}\right)$ contributions. ${ }^{33}$

Thus, the magnetostatic term (in Gaussian units) is generally given by $E_{d i p}=(1 / 2) \int \vec{M}(\vec{r}) \cdot \nabla U d v$, where $U(\vec{r})$ is the magnetostatic potential and $v$ is the particle volume. ${ }^{33} \mathrm{As}$ suming that $\vec{M}(\vec{r})$ varies slowly on the scale of the lattice parameter, we approximate the exchange term by $E_{e x}$ $=A \int \Sigma\left(\nabla m_{i}\right)^{2} d v$, where $A$ is the exchange stiffness constant 

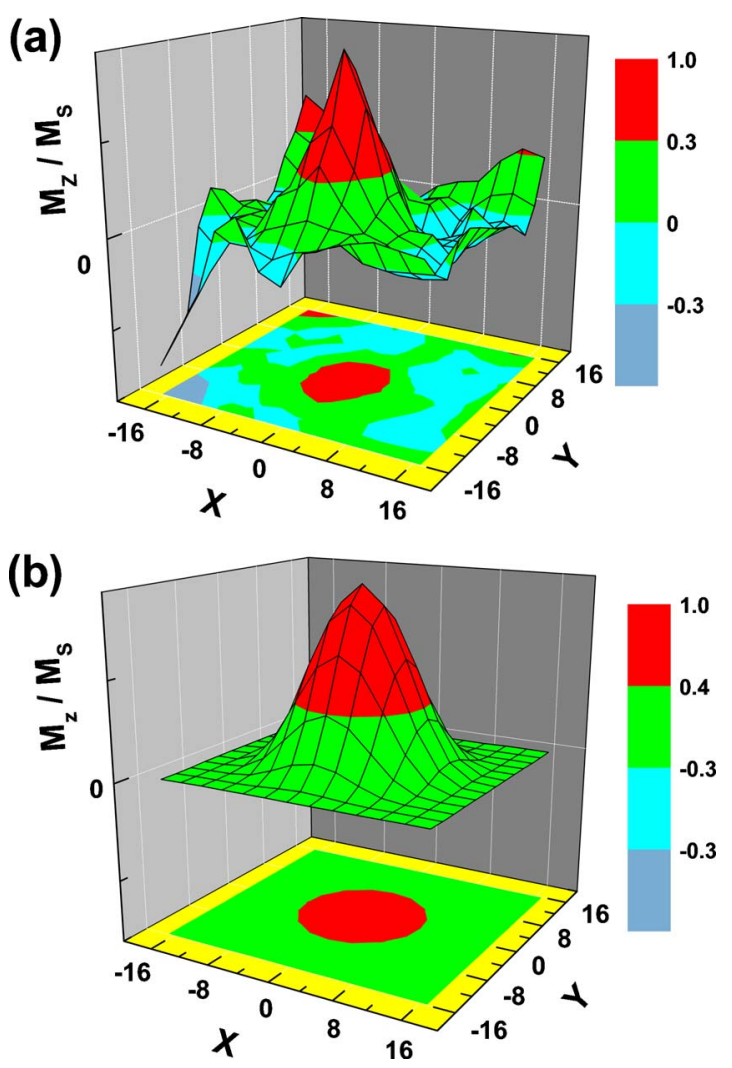

FIG. 7. (Color online) Out-of-plane magnetization (corresponding to the vortex core) in units of $M_{s}$ by using two different theoretical methods: (a) Monte Carlo simulation and (b) analytical calculation using Eq. (5) with $L=20 \mathrm{~nm}$ and $l_{e x}=3.7 \mathrm{~nm}$.

and $m_{i}=M_{i} / M_{0}$ for $i=x, y, z$. We recall that $A$ is proportional to the exchange interaction energy $J$ between the magnetic moments. ${ }^{33}$ The Zeeman term can be evaluated from $E_{z}=-\int \vec{M}(\vec{r}) \cdot \vec{H} d v$ and corresponds to the interaction of the magnetization with an external magnetic field $\vec{H}$. As mentioned above the anisotropy $\left(E_{K}\right)$ contribution is neglected due to the polycrystallinity of the samples. With this procedure, we obtain an analytical expressions for the energy of a noninteracting iron dot of height $L$ and diameter $D=2 R$. For the vortex-core configuration, we assume that the magnetization has the functional form

$$
M(\vec{r})=M_{z}(\rho) \hat{z}+M_{\phi}(\rho) \hat{\phi},
$$

where $\hat{z}$ and $\hat{\phi}$ are unit vectors in cylindrical coordinates and $M_{z}$ and $M_{\phi}$ satisfy the relation $M_{z}^{2}+M_{\phi}^{2}=M_{s}^{2}$. Thus, the profile of the vortex core is fully specified by the function $M_{z}(\rho)$. It is worth noting that the functional form in Eq. (3) does not take into account any dependence of the core shape on the coordinate $z$. Also it does not take account on the "halo" effect at the boundary of the core. ${ }^{34}$ We adopt a vortex-core model ${ }^{25,32}$ with a functional form

$$
M_{z}(\rho)=\left\{\begin{array}{cc}
M_{s}\left[1-(\rho / B)^{2}\right]^{n} & \text { for } 0<\rho \leq B \\
0 & \text { otherwise, }
\end{array}\right.
$$

where $B \leq R$ is a parameter related to the core radius and $n$ is a non-negative constant. Although alternative expressions for
$M_{z}(\rho)$ have been proposed in the literature ${ }^{25,34,35}$ any value of $n \geq 4$ can approximately describe the magnetic vortexcore configuration in nanodots, ${ }^{25,32}$ provided the out-of-plane magnetization at the boundary of the $\operatorname{cor}^{34}$ is small. The total energy, consisting of dipolar and exchange contributions, can be calculated analytically ${ }^{25,32}$ as given, e.g., by Eq. (13) in Ref. 25 for $n=4$. The total energy can be minimized with respect to the parameter $B$ giving $B / l_{e x}=1.83$ $+1.35\left(L / l_{e x}\right)^{0.4}$. Therefore, the vortex-core magnetization [Eq. (4)] is given by the simple expression

$$
M_{z}(\rho)=M_{s}\left\{1-\frac{\rho^{2}}{l_{e x}^{2}}\left[1.83+1.35\left(L / l_{e x}\right)^{0.4}\right]^{-2}\right\}^{4}
$$

which is independent of the dot radius, as pointed out by Shinjo et al. ${ }^{7}$ The above expression for $M_{z}(\rho)$ is consistent with our experiments and simulations. Figure 7(b) shows the vortex-core profile obtained from these analytical calculations with a core radius of approximately $15 \mathrm{~nm}$. This value is in agreement with our experimental and numerical results.

The total magnetization produced by the core region can be obtained by integrating $M_{z}(\rho)$ within the dot volume $v$,

$$
\left\langle M_{z}\right\rangle=\frac{1}{v} \int M_{z}(\rho) \rho d \rho d \phi d z=\frac{B^{2}}{5 R^{2}} M_{s} .
$$

For $L=20 \mathrm{~nm}$ and $l_{e x}=3.7 \mathrm{~nm}$ we obtain $B=16.7 \mathrm{~nm}$ and for $2 R=65 \mathrm{~nm}$ we obtain $\left\langle M_{z}\right\rangle=0.053 M_{s}$ which is close to $0.06 M_{s}$, obtained from our simulations.

\section{Critical diameter for magnetization-reversal mode}

The magnetization reversal in nanodots studied by several groups ${ }^{9,10,36-39}$ showed that for small dots the reversal occurs via coherent rotation, whereas for larger ones it is driven by nucleation, displacement, and annihilation of a single vortex, in agreement with the results discussed above. Also in Ref. 39 , the authors presented a detailed phase diagram showing the critical parameters for stable and metastable spin configurations in a magnetic disk. In this section we describe briefly the crossover between coherent rotation and vortex nucleation in polycrystalline magnetic nanodots. Based on the model of Guslienko et al. ${ }^{10,38}$ for vortex nucleation, we show that below a critical size the reversal process is via coherent rotation and above it vortex nucleation appears. These mechanisms can be investigated by calculating the corresponding nucleation field $\left(\vec{H}_{n}\right)$, defined as the field at which the saturated state becomes unstable and a slight change in the magnetization occurs. ${ }^{33}$ Once the nucleation field for each reversal mode is known, the critical radius at which a vortex nucleates can be obtained.

To calculate the nucleation field for coherent rotation (c), we consider the magnetic energy of a cylindrical dot with uniform in-plane magnetization $\left(\vec{M} / M_{s}=\hat{x} \cos \varphi+\hat{y} \sin \varphi\right)$ at an angle $\varphi$ with the external magnetic field $\vec{H}=\hat{x} H$. In the continuum approach of ferromagnetism, ${ }^{33}$ the magnetic energy can be written in the form

$$
E_{\text {tot }}^{(\mathrm{c})}=-M_{s} H v \cos \varphi+4 \pi M_{s}^{2} v\left(1-N_{z}\right) / 4,
$$

where the first term corresponds to the Zeeman energy and the second one is the dipolar contribution with $N_{z}$ the demag- 
netizing factor. If $H>0$, the energy minimum occurs at $\varphi$ $=0$, corresponding to the magnetization aligned with the applied field. The nucleation field for coherent rotation $\left(H_{n}^{(\mathrm{c})}\right)$ can be obtained as the value of the external field at which $\left(\partial^{2} E^{(\mathrm{c})} / \partial \varphi^{2}\right)_{\varphi=0}=0$, giving $H_{n}^{(\mathrm{c})}=0$.

The vortex nucleation field $\left(H_{n}^{(\mathrm{v})}\right)$ has the form ${ }^{10,38}$

$$
H_{n}^{(\mathrm{v})}=4 \pi M_{s}\left[F(L / R)-4 l_{e x}^{2} / R^{2}\right],
$$

where $l_{e x}=\left(2 A / 4 \pi M_{s}^{2}\right)^{1 / 2}$ is the exchange length, $R$ is the radius, and $L$ is the thickness. The function $F(L / R)$ is given by $F(\beta)=F_{1}(\beta)-F_{2}(\beta)$ with $^{10}$

$$
F_{\mu}(\beta)=\int_{0}^{\infty} \frac{d t}{t}\left[1-\frac{1-e^{-\beta t}}{\beta t}\right] J_{\mu}^{2}(t) .
$$

Thus, by solving $H_{n}^{(\mathrm{v})}=H_{n}^{(\mathrm{c})}$ we can extract information about the critical size for magnetization reversal via coherent rotation and nucleation of a single vortex. Therefore the critical size is given by the relation

$$
F\left(L / R_{n}\right) R_{n}^{2}=4 l_{e x}^{2},
$$

where we define $R_{n}$ as the dot radius such that the nucleation fields for coherent rotation and vortex nucleation are the same. As the nanodots investigated in this paper have the radius larger than their thickness, that is $L / R<1$, we can approximate $F(q)$ by a simple expression $F(q) \approx 0.11 q$ $-0.022 q^{2}$, and Eq. (8) can be expressed as

$$
R_{n} \approx 0.2 L+36.6 l_{e x}^{2} / L,
$$

Therefore, provided the dot thickness $L$ and exchange length $l_{\text {ex }}$ are known, we can estimate $R_{n}$ from Eq. (9). For $R<R_{n}$ we can expect that coherent rotation takes places, whereas for $R>R_{n}$ we can expect that the reversal occurs by nucleation of a single vortex.

The above expression [Eq. (9)] gives insight into the complicated shape of the parameter $\delta$ depicted in Fig. 2. As mentioned earlier, for iron nanodots with $L=20 \mathrm{~nm}$, coherent rotation (hysteresis loops with $\delta \approx 1$ ) for dots with $D$ $\lesssim 60 \mathrm{~nm}$ appears, whereas for larger diameters $(60-65 \mathrm{~nm})$, an increasing number of dots reverse their magnetization through the nucleation of a vortex, and then $\delta$ decreases. The critical diameter at which $\delta$ starts deviating from 1 can be interpreted as $2 R_{n}$. For the parameters used in this paper, $L$ $\approx 20 \mathrm{~nm}$ and $l_{e x} \approx 3.7 \mathrm{~nm}$, we obtain $2 R_{n} \approx 58 \mathrm{~nm}$ in good agreement with the experiments as well as with the Monte Carlo simulations, which averages over a large number of noninteracting nanodots.

\section{CONCLUSIONS}

We report on the dependence of the magnetic state of Fe magnetic nanodots on their diameter, studied with magne- tometry, neutron scattering, numerical simulations, and analytical calculations. This comprehensive study implies that the magnetic reversal for dots smaller than $60 \mathrm{~nm}$ occurs via single-domain state, which is also the ground state at zero field. Their reversal results in the hysteresis loops with parallel branches. When the dot diameter becomes larger than $60 \mathrm{~nm}$, magnetic reversal occurs via nucleation of a vortex which is also the ground state for $65 \mathrm{~nm} \mathrm{Fe}$ nanodots at zero field. This reversal is accompanied by the hysteresis loops with a neck close to zero magnetization. The hysteresis loop shape is characterized by introducing a parameter $\delta$, which measures the ratio of the width of the hysteresis loop at $M$ $=0$ (zero magnetization) and that at $M=0.5 M_{s}$. Using this parameter, we classify different magnetic states as function of dot diameter. In particular, we observe that this parameter is close to one when reversal occurs as a single domain, while it becomes less than one for the vortex state. We find an excellent agreement of the size dependence of this parameter between Monte Carlo simulations and experimental measurements.

For the vortex state, a vortex core, the region with out-ofplane magnetization, appears. Neutron scattering, numerical simulation, and analytic calculations find a core diameter of $16-19 \mathrm{~nm}$ for the $65 \mathrm{~nm}$ magnetic dots. ${ }^{14}$ Monte Carlo simulations and analytical calculations of the vortex core are consistent with a circular Gaussian shape of the core.

\section{ACKNOWLEDGMENTS}

J.M.L., D.A., J.E., and P.L. acknowledge support from AFOSR, FONDECYT under Grants No. 1050066, No. 1080300, No. 11070010, and No. 11080246, the Millennium Science Nucleus "Basic and Applied Magnetism” Grant No. P06-022-F, Financiamiento Basal para Centros Científicos y Tecnológicos de Excelencia, and the program "Bicentenario en Ciencia y Tecnología” PBCT under Project No. PSD-031. J.M.L. acknowledges support from Vicerrectoría Adjunta de Investigación y Doctorado-PUC under Proyecto Límite No. 06/2009. A.H.R. acknowledges support from CONACyT Mexico under Project No. J-59853-F. I.V.R. acknowledges support from Texas A\&M University. I.V.R. and A.H.R acknowledge support from Texas A\&M UniversityCONACyT Collaborative Research Grant Program. X.B. acknowledges the financial support of the Spanish MICINN (Grant No. MAT2009-08667), Catalan DIUE (Grant No. 2009SGR856) and University of Barcelona (International Cooperation). We acknowledge the computer resources from CNS IPICYT, Mexico. Research at UCSD was supported by AFOSR and DOE. 
${ }^{1}$ J. I. Martín, J. Nogues, K. Liu, J. L. Vicent, and I. K. Schuller, J. Magn. Magn. Mater. 256, 449 (2003).

${ }^{2}$ Y. B. Gaididei, V. P. Kravchuk, D. D. Sheka, and F. G. Mertens, Low Temp. Phys. 34, 528 (2008).

${ }^{3}$ J. M. Shaw, S. E. Russek, T. Thomson, M. J. Donahue, B. D. Terris, O. Hellwig, E. Dobisz, and M. L. Schneider, Phys. Rev. B 78, 024414 (2008).

${ }^{4}$ H. Shinohara, M. Fukuhara, T. Hirasawa, J. Mizuno, and S. Shoji, J. Photopolym. Sci. Technol. 21, 591 (2008).

${ }^{5}$ C. Nam, Y. S. Kim, W. B. Kim, and B. K. Cho, Nanotechnology 19, 475703 (2008).

${ }^{6}$ S. Okamoto, N. Nikuchi, T. Kato, O. Kitakami, K. Mitsuzuka, T. Shimatsu, H. Muraoka, H. Aoi, and J. C. Lodder, J. Magn. Magn. Mater. 320, 2874 (2008).

${ }^{7}$ T. Shinjo, T. Okuno, R. Hassdorf, K. Shigeto, and T. Ono, Science 289, 930 (2000).

${ }^{8}$ S.-B. Choe, Y. Acremann, A. Scholl, A. Bauer, A. Doran, J. Stöhr, and H. A. Padmore, Science 304, 420 (2004).

${ }^{9}$ J. Mejía-López, D. Altbir, A. H. Romero, X. Batlle, I. V. Roshchin, C.-P. Li, and I. K. Schuller, J. Appl. Phys. 100, 104319 (2006).

${ }^{10}$ K. Yu. Guslienko, V. Novosad, Y. Otani, H. Shima, and K. Fukamichi, Phys. Rev. B 65, 024414 (2001).

${ }^{11}$ A. Hubert and R. Schafer, Magnetic Domains (Springer-Verlag, Berlin, 2000).

${ }^{12}$ J. P. Park and P. A. Crowell, Phys. Rev. Lett. 95, 167201 (2005).

${ }^{13}$ X. M. Cheng, K. S. Buchanan, R. Divan, K. Y. Guslienko, and D. J. Keavney, Phys. Rev. B 79, 172411 (2009).

${ }^{14}$ I. V. Roshchin, C.-P. Li, H. Suhl, X. Batlle, S. Roy, S. Sinha, M. R. Fitzsimmons, J. Mejía-López, D. Altbir, A. H. Romero, and I. K. Schuller, EPL 86, 67008 (2009).

${ }^{15}$ J. d'Albuquerque e Castro, D. Altbir, J. C. Retamal, and P. Vargas, Phys. Rev. Lett. 88, 237202 (2002).

${ }^{16}$ P. Vargas, D. Altbir and J. d'Albuquerque e Castro, Phys. Rev. B 73, 092417 (2006).

${ }^{17}$ W. Zhang, R. Singh, N. Bray-Ali, and S. Haas, Phys. Rev. B 77, 144428 (2008).

${ }^{18}$ J. Mejía-López, P. Soto, and D. Altbir, Phys. Rev. B 71, 104422 (2005).

${ }^{19}$ C.-P. Li, I. V. Roshchin, X. Batlle, M. Viret, F. Ott, and I. K.
Schuller, J. Appl. Phys. 100, 074318 (2006).

${ }^{20}$ H. Masuda and S. Masahiro, Jpn. J. Appl. Phys., Part 2 35, L126 (1996).

${ }^{21}$ The domain-wall width, $\pi(A / K)^{1 / 2}$, in Fe is estimated to be about $60 \mathrm{~nm}$.

${ }^{22}$ E. C. Stoner and E. P. Wohlfarth, Philos. Trans. R. Soc. London, Ser. A 240, 599 (1948).

${ }^{23}$ R. K. Dumas, C.-P. Li, I. V. Roshchin, I. K. Schuller, and K. Liu, Phys. Rev. B 75, 134405 (2007).

${ }^{24}$ The out-of-plane field is needed to maintain the polarization of the neutron beam and to set the direction of the vortex-core magnetization ("vortex polarity").

${ }^{25}$ D. Altbir, J. Escrig, P. Landeros, F. S. Amaral, and M. Bahiana, Nanotechnology 18, 485707 (2007).

${ }^{26}$ C. A. Ross, M. Farhoud, M. Hwang, H. I. Smith, M. Redjdal, and F. B. Humphrey, J. Appl. Phys. 89, 1310 (2001).

${ }^{27}$ M. Grimsditch, Y. Jaccard, and I. K. Schuller, Phys. Rev. B 58, 11539 (1998).

${ }^{28}$ C. Kittel, Introduction to Solid State Physics, 6th ed. (Wiley, New York, 1986).

${ }^{29}$ K. Binder, Rep. Prog. Phys. 60, 487 (1997).

${ }^{30}$ E. De Biasi, C. A. Ramos, R. D. Zysler, and H. Romero, Phys. Rev. B 65, 144416 (2002).

${ }^{31}$ R. K. Dumas, C.-P. Li, I. V. Roshchin, I. K. Schuller, and K. Liu, Appl. Phys. Lett. 91, 202501 (2007).

${ }^{32}$ P. Landeros, J. Escrig, D. Altbir, D. Laroze, J. d'Albuquerque e Castro, and P. Vargas, Phys. Rev. B 71, 094435 (2005).

${ }^{33} \mathrm{~A}$. Aharoni, Introduction to the Theory of Ferromagnetism (Clarendon Press, Oxford, 1996).

${ }^{34}$ R. Höllinger, A. Killinger, and U. Krey, J. Magn. Magn. Mater. 261, 178 (2003).

${ }^{35}$ See, e.g., N. A. Usov and S. E. Peschany, J. Magn. Magn. Mater. 118, L290 (1993).

${ }^{36}$ R. P. Cowburn, D. K. Koltsov, A. O. Adeyeye, M. E. Welland, and D. M. Tricker, Phys. Rev. Lett. 83, 1042 (1999).

${ }^{37}$ M. Schneider, H. Hoffmann, and J. Zweck, Appl. Phys. Lett. 77, 2909 (2000).

${ }^{38}$ K. Yu. Guslienko and K. L. Metlov, Phys. Rev. B 63, 100403(R) (2001).

${ }^{39}$ S. Savel'ev and F. Nori, Phys. Rev. B 70, 214415 (2004). 